\title{
Wireless Sensor Network as a Tool for Supporting Agriculture in the Precision Irrigation System
}

\author{
Manohar S Chaudhari ${ }^{1}$, Rahul Jaiswal ${ }^{2}$, Chandrakant Birhade ${ }^{3}$, Vishal Bhapkar ${ }^{4}$ \\ Associate Professor, Sinhgad Institute of Technology, Lonavala, Pune, India \\ Department of Computer Engineering, Sinhgad Institute of Technology, Lonavala, Pune, India ${ }^{2,3,4}$
}

\begin{abstract}
Precision irrigation system is basically based on a detailed monitoring of information and data that are necessary for successful decision making in farm production. Wireless Sensor Networks (WSNs) are used for collecting, storing and sharing sensed data. WSN have attracted much attention of researchers in recent years. In past years an extensive research and development work has been done in the field of WSN in the world. WSN have been used for various applications including agriculture, nuclear reactor control, habitat monitoring, environmental monitoring, security and tactical surveillance. WSN have immense potential such that if properly designed and developed can be a solution to a low cost precision irrigation system. The WSN system developed in this paper is for use in precision irrigation system, where real time data of environment and climate are sensed and according to the sensed data a control decisions are taken so that we can modify them. The architecture of WSN consist of a sensor node placed in a field which sends the sensed data to the base station so that a global decision can be taken about the physical environment. This irrigation system promises to give a higher yield and lower input cost by real time monitoring of the field soil and environment conditions using different sensors and thereby improving crop cultivation, reducing time and labor costs.
\end{abstract}

Keywords: Wireless Sensor Network, Precision Agriculture, ZigBee Network, Soil Moisture Sensor.

\section{INTRODUCTION}

WSN is a system comprised of sensors, radio frequency (RF) transceivers, microcontrollers, power sources and base station. Recent development in wireless sensor networking technology have led to the development of low power, low cost, multifunctional sensor nodes. Sensor nodes sense the environment condition and also enable the data processing. Various sensors are used such as soil moisture sensor. They are able to co-ordinate with other sensor in a system and exchange data to the base station. The WSN have many automate devices spread in an environment in order to monitor it and eventually control it, based on the identified physical phenomena. Those devices are called sensors. By using computer resources and appropriate technology, such task are done automatically, easing and utilizing tasks which, a priori, are virtually impossible of full of restrictions when assigned to humans, such as fires identification in forest areas.

WSN can also be deployed where there is ongoing need for environment monitoring and control decision and in tasks that would require too much time and resources if manually done. This way, many applications like electronic commerce, animal tracking and industrial furnaces temperature control need their data to be available at real time. Due to the correctness and consistency of the obtained data, the system is enabled to react in an efficient way.

The different sensors used are soil moisture sensor, light dependent resistor, potentiometer, and thermistor. The moisture sensor uses immersion gold which protects the nickel from oxidation. Electroless nickel immersion gold (ENIG) has several advantages over more conventionalsurface plating such as HASL (solder), including goodoxidation resistance, excellent surface planarity, and usability for untreated contact surfaces such as membrane switches and contact points. Light dependent resistor consist of two cadmium sulphide (cds) photoconductive cells with spectral responses similar to that of the human eye. The cell resistance fall with increasing light intensity. A potentiometer, is a threeterminal resistor with a sliding or rotating contact that forms an adjustable voltage divider. A potentiometer measuring instrument is essentially a voltage divider used for measuring electric potential (voltage). A thermistor is a type of resistor whose resistance varies extensively with temperature. Thermistors are used as flow current limiters, self-resetting overcurrent protectors, temperature sensors, and self-regulating heating elements.

The rest of the paper is organized as follow. Related works are described in section II. The proposed system description, system architecture, and the system performance is reported in section III. Then analysis are reported in section IV to demonstrate the performance of our system. Finally, conclusion are presented in section V.

\section{RELATED WORK}

Simulation allows exhaustive experimenting of the analysed problem. In WSN, additional problems are dealt with in simulations. The sensors behaviour depends upon how they perceive and interact with the environment. In 
many situations, experiments that demand a high number For this application, sensors need to be placed outside, in of sensor nodes have prohibitive costs. In others, like the open field, where power may not be available. So, monitoring volcanic eruptions, the environment will sensors should be battery operated. Sensing location may probably be unreplicable. That is why simulation tools are be identified by integrating GPS with each sensor. useful: they allow the analysed environment to be partially However, that is not a cost effective solution. In this tested by means of virtual sensors. Some simulation tools application, sensors are statically placed at different for wireless sensor networks: J-SIM, SENSE, NetTopo, locations in a field, so the static location of each sensor TOSSIM and TYTHON [1]. Different approaches have with its unique sensor id can be stored in the sensors been explored till date so as to immerge efficient during network configuration and deployment phase.

techniques in terms of conservation of energy resources as well as improving energy efficiency. These approaches can be summed up as, different data prioritization techniques for improving quality-of-services, different routing protocols for secured data collections, different irrigation techniques such as surface irrigation, localized drip irrigation, sprinklers, sub-irrigation. For pest control and fertilizer many methods are used. By providing external energy resources such as solar energy the sensor life time can be improved. We plan to design the irrigation system that will lead to improvement in energy efficiency and conservation of water.

\section{III.PROPOSED WORK}

A precision irrigation system should include following basic functionalities:

- To develop a monitoring system that collects data using a wireless sensor network, and then relays this data through a gateway to a server.

- At the server side the data are stored and analyzed in order to provide the user with useful statistics and alerts so that user can take various decision.

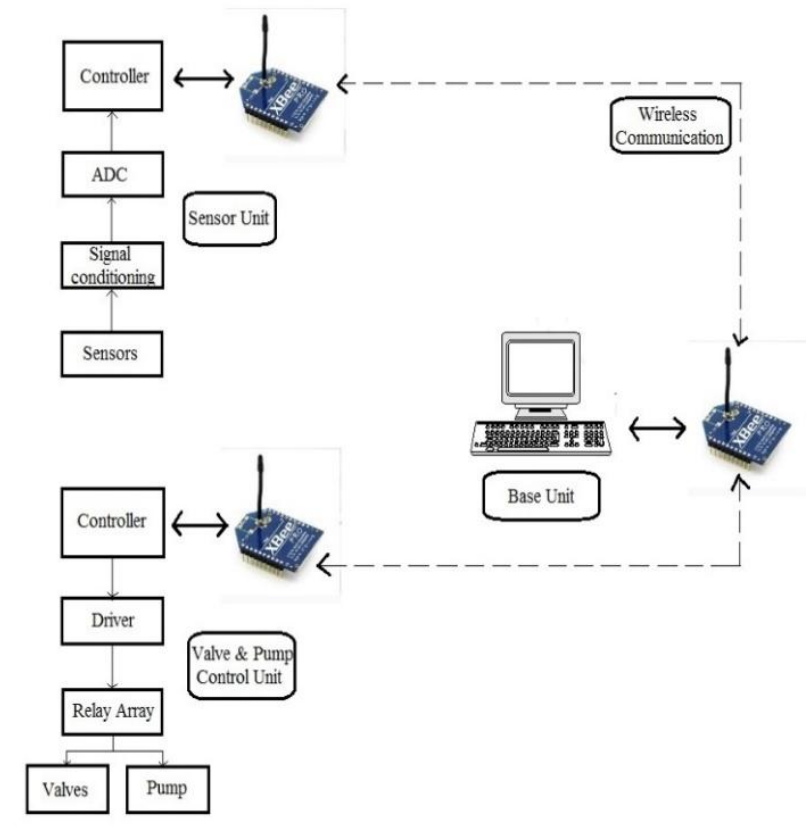

Figure 1: Block Diagram of Proposed System

- The irrigation system should be capable of supporting different types of sensors (for example sensors measuring moisture, temperature, electric potential, light etc.).

In precision agriculture, continuous monitoring of sensor data at every minute may not be always needed. Instead, the data may be monitored on hourly basis or at different times of the day, e.g., morning, noon, afternoon and evening. This, in turn, helps in conserving the battery power of sensor nodes. It is also better to use "sleep and awake" cycle of the wireless sensors judiciously to sense and transmit the sensor data in wake-up phases and put the sensors in sleep mode rest of the time. It may be good idea to aggregate the sensor data captured over a period of time at each node before sending the aggregated data to the monitoring station.

Generally the monitoring station is located far away from the field; therefore, laying wires for transferring sensor data from field to control station is a costly proposition. But the range of battery-operated wireless devices is also limited. So, multi-hop communication is needed to send data to control station. Researchers are now exploring the use of multi-hop wireless sensor network for this purpose. Considering all these functional aspects and limitations in wireless nodes, low power, and low data rate wireless mesh network is found to be a good candidate for realizing the wireless sensor network.

\section{A. Irrigation Station}

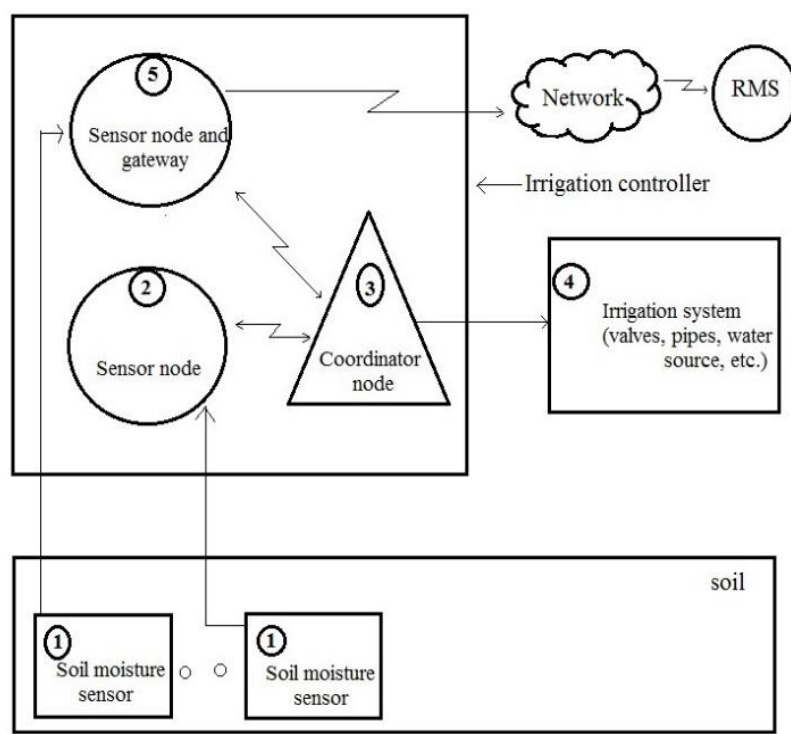

Figure 2: The architecture of irrigation station The workflow of the precision irrigation system can be mapped by a component system architecture depicted in figure 2 which includes various component such as soil moisture sensor, coordinator node, sensor node, gateway node, and irrigation system. 
B. In-Field Sensor Node

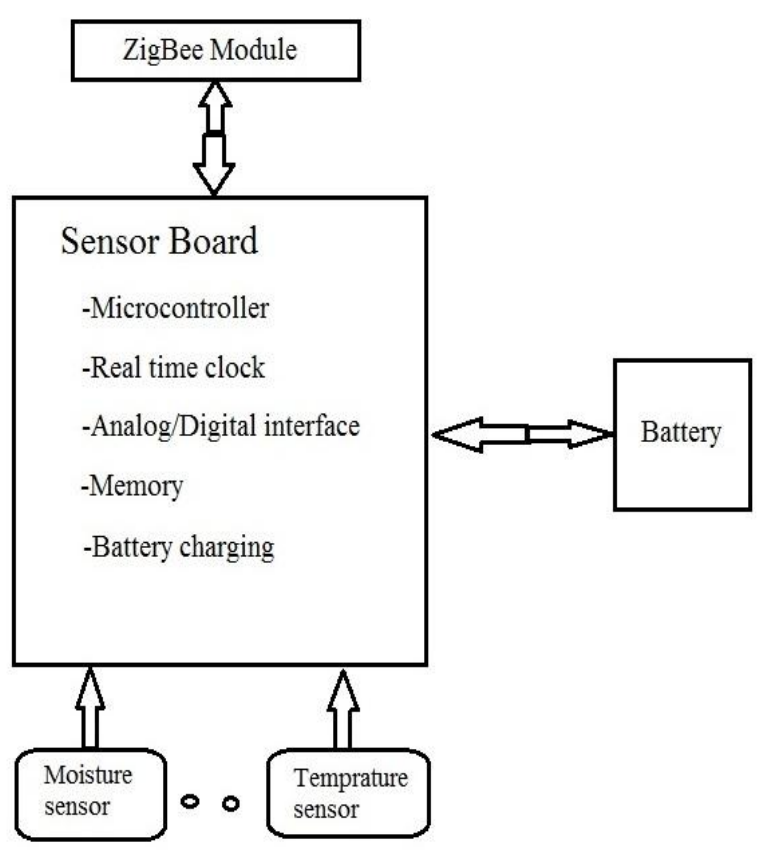

Figure 3: The architecture of in-field sensor node

Figure 3 shows the architecture of an in-field sensor. This sensors are equipped with a ZigBee module for communication with the base station.

A program is developed and uploaded to measure soil moisture, their battery levels, and soil temperature. Once the measurements are completed, the sensed data is forwarded through ZigBee transceivers to the base station for processing.

\section{Actuator Node}

The precision irrigation system consist of four components: solenoid valves; latching circuit; drip pipes; and powering system. It is necessary to use a latching circuit as a means of saving energy. Continuously sending and holding the pulse for entire irrigation period could waste battery power.

The latching circuit make use of a short pulse from I/O pins of the MCU. The latching circuit consist of digital NAND gates, optocouplers, power transistors, and switching transistors. Power transistors are used to switch on or off solenoid valves. Switches are included in the latching circuit to allow manual opening and closing of the valves in case of emergency.

It was essential to use solenoid valves because of the low power consumption, low cost, and the possibility of using a $12 \mathrm{~V}$ direct current power supply. With this arrangements, the base station is able to control the irrigation system by sending short pulses to its microcontroller unit $\mathrm{I} / \mathrm{O}$ pins.

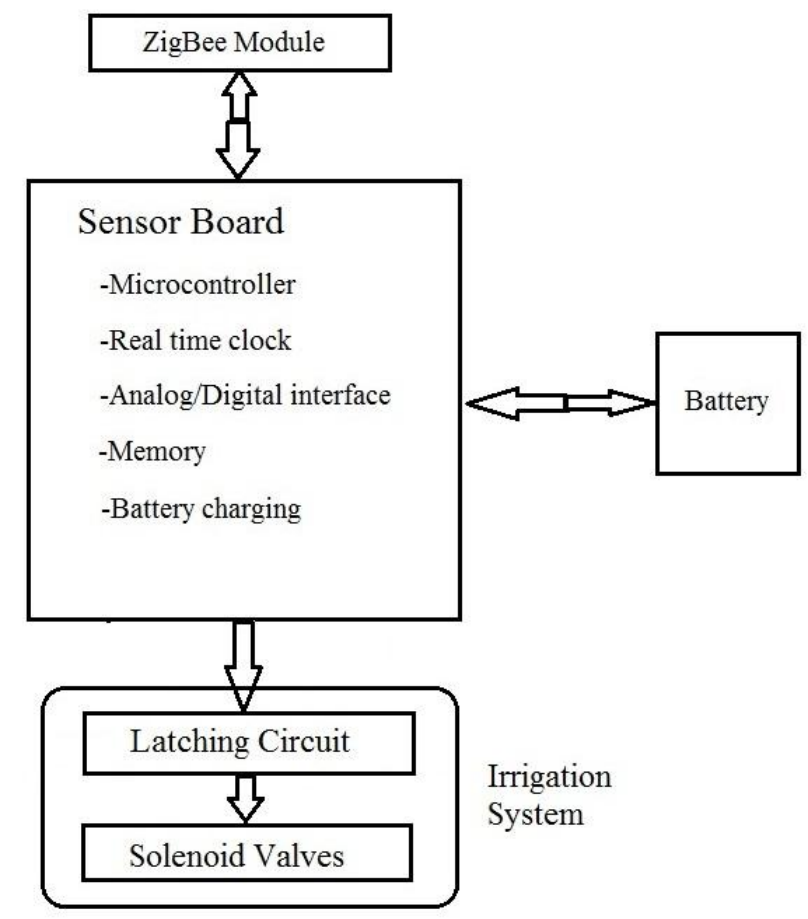

Figure 4: Actuator node

\section{IV.ANALYSIS}

Deploying the system developed with help of thesis presented above, we may improve farming practice and hence enhance the production. With the automation of the farming process, completely relying on man power for farming will not be necessary. The proposed system will make our system energy efficient. The kind of irrigation system we plan to design would result in conservation of water and would avoid wastage of fertilizers. Drip irrigation for fertilizers in liquid state as well as watering the field, and sprinklers for application of pesticides. Considering the present available techniques which are being used for farming, the system built from the above idea presented will surely enhance the outcome of the field.

\section{Conclusion}

A sensing system combined with ZigBee based wireless networking has been tested to be quite effective and efficient. Precision irrigation system and WSN applications combine an exciting new area of research that will greatly improve quality in precision irrigation, agricultural production and will have reduction in cost. Besides, the ease of deployment and system maintenance, monitoring opens the way for the acceptation of WSN systems in precision irrigation. We have tried to lower implementation cost and thus make WSN a more appealing solution for all kinds of fields. The applications of ZigBee wireless technology in agriculture can be extended to automated irrigation control, real time field monitoring, and remote operation of field machinery. When applied to a plot of farm, the goal is to identify areas with low humidity levels, areas which require fertilization and areas which are relatively arid, 
through data captured by sensors that are geographically distributed and irrigate and/or fertilize only those areas, saving resources and avoiding water wastage and threat of soil salinity, when applied to a real situation.

Thus, we may conclude that wireless sensor network when applied in a systematic way, may prove to be a boon for agriculture field.

\section{REFERENCES}

[1] Gracon H. E. L. de Lima, Lenardo C. e Silva, Pedro F. R. Neto, "WSN as a Tool for supporting Agriculture in the Precision Irrigation" 2010 Sixth International Conference on Networking and Services, DOI 10.1109/CyberC.2010.36.

[2] Daozong Sun, Sheng Jiang, Jingchi Tang, Weixing Wang (correspondence author) "WSN Design and Implementation in a Tea Plantation for Drought Monitoring" 2010 International Conference on Cyber-Enabled Distributed Computing and Knowledge Discovery, DOI 10.1109/CyberC.2010.36.

[3] Manijeh Keshtgari, Amene Deljoo, "A Wireless Sensor Network Solution for Precision Agriculture Based on ZigBee Technology" Wireless Sensor Network, 2012, 4, 25-30.

[4] N. Wang, N. Zhang and M. Wang, "Wireless Sensors in Agriculture and Food Industry: Recent Development and Future Perspective," Computers and Electronics in Agriculture Journal, Vol. 50, 2006, pp. 114-120.

[5] Million Mafuta, Marco Zennaro, Antoine Bagula, Graham Ault, Harry Gombachika, and Timothy Chadza, "Successful Development of a Wireless Sensor Network for Precision Agriculture in Malawi" International Journal of Distributed Sensor Networks Volume 2013, Article ID 150703, 13 pages.

[6] Sherine M. Abd El-kader, Basna M. Mohammad El-Basioni, "Precision farming solution in Egypt using the wireless sensor network technology” Egyptian Informatics Journal (2013) 14, 221-233.

[7] S. Blackmore, "Precision Farming: An Introduction," Outlook on Agriculture Journal, Vol. 23, 1994, pp. 275-280.

[8] A. Baggio, "Wireless Sensor Networks in Precision Agriculture," Delft University of Technology, Delft, 2009.

[9] M. Keshtgary and A. Deljoo, "AN efficient wireless sensor network for precision agriculture," Canadian Journal on Multimedia and Wireless Networks, Vol. 3, no. 1,pp. 1-5, 2012

[10] W. Cao, G. XU, E. Yaprak, R. Lockhart, T. Yang and Y. Gao, "Using Wireless Sensor Networking (WSN) to Manage MicroClimate in Greenhouse," Manufacturing Enterprise Solutions Associations, Vol. 17, No. 3, 2008, pp.636-641.

[11] Y. Zhou, X. Yang, L. Wang, and Y. Ying, "A wireless design of low-cost irrigation system using ZigBee technology," in Proceedings of the International Conference on Networks Security, Wireless Communications and Trusted Computing (NSWCTC'09), pp. 572-575,April 2009.

[12] Xin Dong, Mehmet C. Vuran, and Suat Irmak, "Autonomous precision agriculture through integration of wireless underground sensor networks with center pivot irrigation systems," Ad Hoc Networks, Volume 11, Issue 7, September 2013, Pages 1975-1987, DOI:10.1016/j.adhoc.2012.06.012 